\title{
En búsqueda de ciudades sostenibles, seguras e inclusivas. Aportes críticos desde la ergonomía y el diseño
}

\author{
Carlos Aceves-González ${ }^{1}$ | John Rey-Galindo 2 \\ Recibido: 26-09-2020 | Versión final: 31-01-2021
}

Resumen

\begin{abstract}
Actualmente existe evidencia que sugiere la urgencia de avanzar en el (re)diseño y construcción de espacios urbanos que consideren las necesidades y limitaciones de los ciudadanos y que reduzcan el impacto al ambiente. La Ergonomía (o Factores Humanos) y el Diseño Centrado en el Usuario, como disciplinas enfocadas en el análisis de las interacciones del ser humano con su entorno, tienen el potencial para asegurar que todo artefacto, espacio o servicio sea diseñado de acuerdo a las características y necesidades humanas y con ello asegurar un mejor funcionamiento de los sistemas. Este artículo tiene como fin presentar y describir el uso de principios, métodos y herramientas de la Ergonomía y el Diseño Inclusivo y Diseño de Servicios para el análisis de sistemas urbanos (particularmente sistemas de movilidad) que pueden aportar, desde su visión centrada en el ser humano y su naturaleza sistémica, al diseño de ciudades más sostenibles, inclusivas y seguras. Se describe al final, parte del desarrollo y la aplicación de la herramienta blueprint de servicios inclusivos como estudio de caso para el análisis del transporte público en el Área Metropolitana de Guadalajara, México.
\end{abstract}

Palabras clave: Diseño inclusivo; diseño de servicios; blueprint, diseño centrado en las personas

Citación

\section{In Search of Sustainable, Safe and Inclusive Cities. Critical Contributions from Ergonomics and Design}

Abstract

\begin{abstract}
There is currently evidence suggesting the urgency of advancing in the (re)design and construction of urban spaces that consider the needs and limitations of citizens and reduce the impact on the environment. Ergonomics (or Human Factors) and User-Centred Design, as disciplines focused on the analysis of human interactions with their environment, can ensure that any artefact, space or service is designed according to the characteristics and human needs and thereby provide better functioning of the systems. This paper aims to present and describe the use of principles, methods and tools of Ergonomics and Inclusive Design and Service Design to analyse urban systems (particularly mobility systems). This discipline and design approaches can contribute, from their human-centred vision and systemic nature to the design of more sustainable, inclusive and safe cities. At the end of the paper, it is described, part of the development and application of the inclusive service blueprint tool as a case study to the analysis of public transport in the Metropolitan Area of Guadalajara, Mexico.
\end{abstract}

Keywords: Inclusive design; service design; blueprint, human-centred design

${ }^{1}$ Psicólogo, Ph.D. en Ergonomía y Diseño de Servicios Inclusivos por la Universidad de Loughborough, Reino Unido. Director del Centro de Investigaciones en Ergonomía, Universidad de Guadalajara, Guadalajara, México (ORCiD: 0000-0002-6720-808X, WoS Researcher ID: $\underline{\mathrm{H}-8138-2018)}{ }^{2}$ Diseñador Industrial, Maestro en Diseño Industrial con orientación en Ergonomía por la Universidad Nacional Autónoma de México, UNAM. Profesor Asociado Centro de Investigaciones en Ergonomía, Universidad de Guadalajara, Guadalajara, México (ORCiD: 0000-0002-0458-7107). Correo de contacto: c.aceves@academicos.udg.mx 


\section{Introducción}

El desarrollo sostenible es quizás el tema más importante de la actualidad por sus implicaciones para el bienestar y la calidad de vida de estas y las generaciones venideras. La necesidad de redireccionar la manera en la que vivimos los seres humanos se hace cada vez más evidente e importante a causa de fenómenos como el cambio climático o las paupérrimas condiciones de vida que experimentan comunidades enteras.

Es en el marco del Reporte Brundtland (WCED, 1987) titulado "Our Common Future" donde se propone la sostenibilidad como ruta de desarrollo de las sociedades del mundo. Este reporte, marca una búsqueda más global para encontrar formas de desarrollo que disminuyan el impacto que nuestros modos de vida tienen sobre el medio ambiente. A lo largo del tiempo, la sostenibilidad como concepto se ha concentrado en las dimensiones ambiental, social y económica (Kuhlman \& Farrington, 2010) como sus grandes pilares de avance, alrededor de los cuales se conjuntan sus grandes esfuerzos.

El mismo Reporte Brundtland reconoció a las ciudades como escenarios de gran importancia. Estos grandes puntos de concentración de las interacciones humanas juegan un papel central y requieren de una clara revisión de la manera en cómo operan. Según algunos pronósticos, para el año 2050, más del 70\% de la población del mundo se encontrará viviendo en las ciudades (Roth, 2012). Esto representa enormes retos en la manera en cómo se piensa el crecimiento de estos macro-sistemas, mismos que hoy por hoy están ya marcados por múltiples problemas.

Considerando las tres esferas de la sostenibilidad, el listado de problemas en las ciudades es extenso. El intensivo consumo de energía con un crecimiento económico insostenible, alto volumen de tráfico, redes de transporte saturadas, contaminación del aire y el agua (ya para el 2005 las ciudades producían el $80 \%$ de los gases de efecto invernadero y consumían el 75\% del agua en el mundo), desechos tóxicos, falta de recursos, inequidad y vulnerabilidad social evidente en la falta de infraestructura para ampliar el acceso a servicios públicos, reducción de la salud pública, falta de políticas (Bibri \& Krogstie, 2017; Keivani, 2010) son algunos de los problemas más visibles en las ciudades.

A lo anterior se debe sumar que, como todo sistema complejo, el contexto particular define el comportamiento de los fenómenos, por lo que la sola manera de estudiar y comprender lo que pasa, requiere de perspectivas diversas (Habib \& Shokoohi, 2009). Esto se convierte en un reto aún mayor cuando además de comprender los fenómenos, se tiene la tarea de re-direccionar el camino para encontrar soluciones. Basta con revisar escenarios como los de las ciudades latinoamericanas, para identificar la magnitud de la diversidad del fenómeno y sus implicaciones.

Así como se reconoce la ciudad como un mecanismo complejo y problemático, debe ser entendido también como un agente de cambio, en donde la sostenibilidad dependerá de la manera en cómo se concibe el modelo de urbanización (Roth, 2012). Es necesario entonces, tener una clara perspectiva a mediano y largo plazo de las formas de construcción, infraestructura, operación y función de las ciudades (Bibri \& Krogstie, 2017).

En relación a lo anterior, iniciativas como la Agenda 2050 para el Desarrollo Sostenible de la Organización de la Naciones Unidas (ONU), constituyen una referencia, tanto de los aspectos a considerar para el futuro de ciudades más saludables y adecuadas, como del rol que tienen estos sistemas sostenibles dentro del plan de desarrollo mundial como uno de sus 17 objetivos. El propósito establecido por la ONU, se enfoca en la transformación de las ciudades y en general de los asentamientos humanos, en espacios inclusivos, seguros, resilientes y sostenibles.

ACE, 16 (4.7) CC BY-ND 3.0 ES | UPC Barcelona, España | Título del artículo escrito en mayúsculas y minúsculas, si es demasiado largo debe ajustarlo a dos líneas como máximo. DOI: http://dx.doi.org/10.5821/ace.16.47.9691 
Esta transformación de los espacios habitables, que se hace cada vez más necesaria, requiere de nuevas formas de entender los procesos de cambio. Para ello, es esencial considerar como centro del proceso el bienestar del ser humano y del medio ambiente. Basados en esta idea, este artículo tiene como objetivo presentar aportes para el diseño de ciudades más inclusivas, seguras y sostenibles, sugiriendo como estrategia el uso de la disciplina científica de la ergonomía, con su enfoque centrado en las personas y en las actividades humanas; el diseño inclusivo con su filosofía de considerar las capacidades y limitaciones, así como las necesidades y expectativas de los diversos usuarios; y el diseño de servicios con su perspectiva de análisis de la interacción de los componentes de los sistemas. Para finalizar, se presenta parte del análisis del transporte público en el Área Metropolitana de Guadalajara, como estudio de caso en el que se aplican conceptos y herramientas de esta disciplina y enfoque de diseño. Consideramos que estos aportes pueden traer nuevos aires en el proceso de desarrollo y mejora de las ciudades.

\section{La Ergonomía, disciplina centrada en las personas que contribuye en la construcción de ciudades más sostenibles}

La ergonomía se define como la disciplina científica que se encarga de comprender las interacciones entre los seres humanos y los elementos de un sistema y la profesión que aplica teoría, principios, datos y métodos para diseñar con el fin de optimizar el bienestar humano y el rendimiento general del sistema (IEA, 2000). Si bien, a la ergonomía se le conoce desde otros ámbitos, en el mejor de los casos, como una disciplina enfocada en los sistemas productivos, (de allí la etimología de la palabra ergon = trabajo, nomos = leyes), su enfoque en el análisis de la interacción entre los componentes de los sistemas laborales, abrió el espacio para evidenciar su potencial como herramienta de transformación de otros sistemas (Wilson, 2000).

Esta evolución es clara en tres aspectos que asoman en la definición de la International Ergonomics Association (IEA) que aparece en el párrafo anterior. El primero se refiere a su carácter de disciplina con una filosofía y enfoque de sistemas; el segundo aspecto es el bienestar humano como uno de los grandes objetivos, y el tercero se refiere a que usa el pensamiento de diseño como vía para la transformación de los sistemas que analiza e interviene.

El enfoque sistémico de la ergonomía le permite analizar y entender las problemáticas desde las características de la interacción de los componentes, con el fin de identificar de manera más clara las implicaciones que dichas interacciones tienen sobre el desempeño del sistema. Wilson (2014) ha reconocido que la noción de sistema de la ergonomía se define por seis aspectos básicos que son: a) Enfoque sistémico, que le permite un acercamiento a la complejidad de la problemática a partir del estudio de las relaciones de los componentes en sus distintas escalas. Es lo que denomina un sistema de sistemas. b) Contexto, que se refiere a la importancia de entender las problemáticas en sus escenarios reales. Esto prioriza el trabajo en campo por sobre el trabajo en laboratorio, para la ergonomía. c) Interacciones, que se constituyen en uno de sus puntos centrales de análisis y que contempla aspectos entre los que están el ser humano, aspectos técnicos, información y componentes sociales, políticos, económicos y organizacionales. Todo ello en distintas escalas y en distintos momentos del sistema. d) Holístico, que se refiere a su capacidad de entender los fenómenos desde una perspectiva amplia, basado en su capacidad para entender a los seres humanos desde los diversos aspectos que están implícitos en las interacciones dentro del sistema, como sus características y limitaciones físicas, cognitivas y sociales. e) Emergente, que se refiere a su capacidad para el reconocimiento y ajuste a condiciones cambiantes en los sistemas. Esto se debe a que la ergonomía trata con escenarios con usuarios, entornos y situaciones reales, muchas veces en contextos pobremente diseñados. f) Embebido, que hace referencia a la capacidad del ergónomo

ACE, 16 (47) CC BY-ND 3.0 ES | UPC Barcelona, España | Título del artículo escrito en mayúsculas y minúsculas, si 3 es demasiado largo debe ajustarlo a dos líneas como máximo. DOI: http://dx.doi.org/10.5821/ace.16.47.9691 
de acercarse de manera profunda a las organizaciones o los sistemas, trabajando desde una perspectiva participativa en donde se involucran los diferentes actores.

El segundo punto de la definición de la IEA que resulta esencial, se refiere a entender a la ergonomía en su condición de disciplina centrada en el ser humano. La ergonomía desde su surgimiento y a lo largo de la historia ha concentrado sus métodos de análisis y desarrollo en el bienestar del ser humano y el desempeño del mismo dentro de los sistemas. Dul et al. (2012) mencionan, con relación al bienestar, que aspectos como la salud, seguridad, satisfacción, comodidad, aprendizaje y desarrollo personal, han sido líneas de acción de la disciplina. De igual manera, respecto al desempeño, temas como productividad, eficiencia, eficacia, calidad, innovación y flexibilidad, han tenido espacios centrales para la ergonomía.

La naturaleza de la ergonomía como disciplina centrada en el ser humano, le ha permitido también una comprensión más amplia de las características de los seres humanos y las implicaciones que ellas tienen en su interacción con los demás componentes de los sistemas. De allí se desprende su necesidad por comprender la diversidad de características de las personas, que se hace evidente en aspectos como su anatomía, antropometría, biomecánica, fisiología, sumadas a aspectos económicos, sociales y culturales.

El tercer aspecto de la definición de la IEA, es la relación tácita de la ergonomía con el diseño. Si bien, en muchos casos a la ergonomía y a las áreas del diseño se les puede reconocer como campos del conocimiento separados, es su conjunción la que permite encontrar contextos o sistemas más coherentes con los seres humanos. En un trabajo realizado por Wogalter, Hancock y Dempsey (1998), en donde buscaban caracterizar a la ergonomía y su campo de acción, encontraron como uno de los puntos más relevantes, que la palabra que se encuentra más presente en las definiciones de ergonomía, después de la palabra humano, es la palabra diseño. Esto hace evidencia de la relación que mantiene la ergonomía con las disciplinas proyectivas siendo estas últimas el vehículo más idóneo para intervenir y contribuir en la mejora de los sistemas de interés de la ergonomía.

El aspecto sistémico, su naturaleza de disciplina centrada en el usuario y su estrecha relación con el diseño, han contribuido al acercamiento de la ergonomía a las problemáticas presentes en la sostenibilidad. En este sentido, Moray (1995) reconoció que una visión ampliada de sistemas que incluyera aspectos contextuales y culturales, sumado al reconocimiento de los llamados que se hacían desde otras áreas como el diseño, para lograr entornos con un mayor bienestar humano y ambiental, serían factores esenciales para entender el rol de la ergonomía en los retos que estarían presentes en el siglo XXI, marcados, en especial, por la necesaria mirada a la sostenibilidad.

La ergonomía entonces, comenzó a sumarse a los esfuerzos por definir un mundo más sostenible. Para ello, a lo largo del tiempo se han encontrado evidencias de cómo las intervenciones ergonómicas presentan una relación directa no solo con el bienestar de las personas, sino que contribuyen a pensar en sistemas más adecuados en el manejo de los recursos y el medio ambiente (Haslam \& Waterson, 2013). El diseño de productos orientados a la sostenibilidad y el bienestar de los usuarios; el análisis del trabajo en sus relaciones con el medio ambiente y el bienestar, a través de las prácticas más sostenibles de empleo; el diseño de sistemas de trabajo más eficientes (Thatcher, 2013), entre otros, se han planteado como áreas de desarrollo de la ergonomía dentro de la sostenibilidad. A lo anterior se suma una visión más holística de los fenómenos que atañen a la sostenibilidad y la integración en ella de temas del núcleo de la ergonomía como el funcionamiento de las organizaciones y los recursos humanos (Haslam \& Waterson, 2013).

Respecto a las ciudades y la necesidad de trabajar en su desarrollo sostenible, la ergonomía también ha comenzado a dar algunas luces. Respecto a los problemas que Moray (1995) consideró como

ACE, 16 (4.7) CC BY-ND 3.0 ES | UPC Barcelona, España | Título del artículo escrito en mayúsculas y minúsculas, si es demasiado largo debe ajustarlo a dos líneas como máximo. DOI: http://dx.doi.org/10.5821/ace.16.47.9691 
claves para el desarrollo de la ergonomía dentro de la sostenibilidad, uno de los que parece haber despertado más el interés para los ergónomos es lo concerniente a la urbanización. En este sentido, Thatcher et al. (2018) reportan algunos planteamientos teóricos enfocados en los posibles aportes de la ergonomía en el diseño de ambientes urbanos.

Un ejemplo de lo anterior es el planteamiento de Richardson et al. (2017) que se vale de la teoría de la relación humano-naturaleza en la que se plantea la búsqueda del bienestar humano, (tema esencial de la ergonomía), a través del uso de una conexión constante y directa con la naturaleza. Esta idea plantea, basada en evidencias, que volver a integrar espacios naturales en espacios como las ciudades no solo tiene impactos positivos en el estado físico y mental de los habitantes, sino que los hace más conscientes del valor del mundo natural y en ese sentido mejora sus comportamientos en relación con su cuidado.

En un sentido similar, Wolf (2003) quien se enfoca en la importancia de la implementación de infraestructura verde en las ciudades para mejorar las condiciones socio-ambientales, pone sobre la mesa también la importancia de entender de qué manera estos cambios repercuten en aspectos psicosociales. Así pues, propone, como un factor esencial en el proceso de planeación sostenible, la inclusión de la ergonomía para comprender y con ello mejorar los aspectos relacionados con el bienestar humano.

Algunos de los beneficios de la aplicación de la ergonomía en el mejoramiento de las ciudades, se manifiestan en un sentido menos evidente. Sin embargo, se ha logrado encontrar que las mejoras ergonómicas tienen un impacto efectivo. Esto se puede explicar, por ejemplo, cuando se cuenta con un servicio público de transporte que puede ser fácilmente usado, pero que además resulta en una experiencia agradable para las personas. Un sistema de esas características puede hacer más factible que se desincentive el uso del vehículo particular por parte de otros usuarios, lo que traerá una disminución de consumo de energía, pero también de agentes contaminantes.

Si bien se ha trabajado en la aplicación de la ergonomía en el diseño de las ciudades, aún es poca la evidencia de su presencia en estos procesos, lo que ha ralentizado la comprensión de su importancia (Thatcher et al., 2018). En Latinoamérica (y probablemente sea el caso de muchas ciudades en países de bajo y mediano ingreso) se encuentra aún menos evidencia de la presencia de la ergonomía en el diseño de ciudades. Factores como la falta de una mirada sistémica de los procesos de mejora y de procesos centrados en el usuario; o visiones limitadas o sesgadas en las políticas públicas (CEPAL, 2010) pueden ayudar a entender este fenómeno.

Más allá de las razones de la falta de una visión ergonómica en el desarrollo de políticas sostenibles en el contexto Latinoamericano, se debe reconocer la importancia que esta perspectiva tendría en el combate de fenómenos como la extrema desigualdad social, que es la más alta del mundo según cifras de referencia internacionales y que se debe, entre otras cosas, a las formas de urbanización con un enfoque periférico que produce segregación social con el favorecimiento de la aparición de asentamientos informales, falta de servicios públicos, gentrificación y problemas de movilidad, entre otros (Calderón, Salas y Ávila, 2020; Iracheta, 2020; Jordán, Riffo y Prado, 2017).

A un contexto desigual marcado por carencias de una infraestructura limpia, incluyente y segura (CEPAL, 2010), debemos sumar el alto y negativo impacto que han tenido las formas de crecimiento de las ciudades en el manejo del entorno natural, a partir de una idea globalizada de progreso que ha ignorado por completo la diversidad de características y requerimientos de las comunidades y el entorno en cada una de las ciudades latinoamericanas, provocando, además de muchos otros fenómenos, la elevada contaminación del aire y el agua (Winchester, 2006) que tanto afectan a la población de esta parte del mundo.

ACE, 16 (47) CC BY-ND 3.0 ES | UPC Barcelona, España | Título del artículo escrito en mayúsculas y minúsculas, si es demasiado largo debe ajustarlo a dos líneas como máximo. DOI: http://dx.doi.org/10.5821/ace.16.47.9691 
Así pues, se hace importante la búsqueda de alternativas que permitan que cada vez más, la ergonomía sea integrada en los procesos de diseño de las ciudades con características similares a las que se tienen en Latinoamérica; máxime, cuando cada vez es más evidente que el camino hacia un desarrollo sostenible pasa por garantizar el bienestar del ser humano. Es importante insistir en que la naturaleza sistémica de la ergonomía aunada a su enfoque centrado en el ser humano y su conexión directa con las áreas del diseño, la convierten cada vez más en un campo fértil; impulso de nuevas maneras de entender el desarrollo humano y en ese sentido en un aliado esencial para conseguir ciudades con mejores condiciones de vida.

\section{Necesidad de ciudades inclusivas}

Un postulado simple de este texto parte de la idea fundamental del enfoque de Diseño Inclusivo: "El diseño inclusivo es un mejor diseño" (Clarkson et al., 2007). Esta idea, por tanto, pone énfasis en la aplicación de la filosofía para la inclusión a través del proceso de diseño. Sobre esta base, este apartado se construye considerando que "las ciudades inclusivas son mejores ciudades". Por mejores ciudades léase aquellas que son más seguras, resilientes y sostenibles

Actualmente nos encontramos en un contexto marcado por el envejecimiento de la población, mismo en el que se destaca la necesidad de ayudar a las personas a envejecer bien, mediante la provisión de ambientes que les brinden apoyo y les faciliten realizar sus actividades. Las Naciones Unidas (United Nations, 2002) argumentan que el envejecimiento de la población es el proceso por el cual las personas mayores se convierten en una porción proporcionalmente mayor del total de la población. Los datos de esta organización indican que en 2009 había un estimado de 737 millones de personas de 60 años o más. Sin embargo, se prevé que este número aumente a 2 mil millones en 2050, momento en el que se espera que haya más personas mayores de 60 que niños menores de 14 años (United Nations, 2009).

Sin duda, existen desde hace algunas décadas diversos esfuerzos para avanzar en el diseño de productos, espacios y entornos urbanos más amigables con las personas mayores - especialmente en los países de mayor ingreso -. Sin embargo, el acceso a espacios al aire libre y los edificios aún no están totalmente garantizados para personas con diferentes necesidades, capacidades y limitaciones. Según la Organización Mundial de la Salud (OMS) (WHO, 2007) tanto en países desarrollados como en países en desarrollo, la gente piensa que su ciudad no fue diseñada para personas mayores y también informan que la prestación de servicios comerciales y públicos presentan problemas para satisfacer las necesidades de este grupo de personas. Los datos de dicho informe sugieren que, junto con la necesidad de un entorno construido accesible, existe también una necesidad imperante de servicios que puedan ser utilizados por una gama más amplia de usuarios.

Las iniciativas de diseño para la inclusión han sido impulsadas por el incremento de la población de adultos mayores; en conjunto con el reconocimiento de los derechos de personas con alguna discapacidad a través de imperativos legislativos como la Americans with Disabilities Act (ADA, 1990) establecida en Estados Unidos y la Disability Discrimination Act (DDA, 1995) en el Reino Unido (ahora The Equality Act, 2010) (Coleman et al., 2003). En este contexto, surgieron algunos enfoques de diseño con la intención de brindar beneficios a una mayor cantidad de usuarios, en particular a personas con alguna discapacidad y/ o adultos mayores (Clarkson et al., 2003). Clarkson y Coleman (2013) argumentan que estos cambios legislativos y enfoques de diseño han cambiado progresivamente el lenguaje académico de un enfoque hacia el ellos - ancianos y discapacitados - a uno hacia el nosotros. El proceso de envejecimiento suele estar relacionado de alguna manera con la discapacidad, debido a que se acepta que el deterioro físico y psicológico se vuelve más probable a medida que aumenta la edad (Smith, 1990). De hecho, la mayoría de las investigaciones sobre la salud de las personas

ACE, 16 (4.7) CC BY-ND 3.0 ES | UPC Barcelona, España | Título del artículo escrito en mayúsculas y minúsculas, si es demasiado largo debe ajustarlo a dos líneas como máximo. DOI: http://dx.doi.org/10.5821/ace.16.47.9691 
mayores se han centrado en las tendencias hacia la discapacidad (Crimmins, 2004). Según la OMS (WHO, 2011), la relación es sencilla: existe un mayor riesgo de discapacidad a edades más avanzadas. Los datos muestran que la prevalencia de la discapacidad en la población global es inferior al 20\% para las personas de 45 a 54 años y aumenta a más del 50\% para las personas de 75 años o más. Una mayor tasa de discapacidad entre las personas mayores es el resultado de una acumulación de riesgos para la salud a lo largo de la vida debido a enfermedades, lesiones y enfermedades crónicas, sin embargo, una persona mayor puede sufrir alguna discapacidad solo por el proceso natural de envejecimiento mismo que reduce sus capacidades.

Se debe considerar que la discapacidad es un espectro, en tal caso todos nosotros podríamos ser considerados discapacitados. Decidir hasta qué punto la capacidad debe verse afectada para constituir una discapacidad no es un asunto fácil (The Lancet, 1999). Es importante señalar que, aunque el envejecimiento puede traer consigo una disminución de capacidades, no puede ni debe ser visto como sinónimo de discapacidad. La discapacidad desde un enfoque social y no médico, se entiende como el producto de unas malas condiciones del sistema, el cual no responde a los requerimientos y características de las personas, para que puedan llevar a cabo sus actividades de manera adecuada.

La OMS que además de los impedimentos físicos, también incluye limitaciones en actividades como la incapacidad para leer y restricciones en la participación, como la exclusión de la escuela o el trabajo (WHO, 2012). La OMS reconoce que la discapacidad no se puede explicar solo por las limitaciones de las personas, sino que se debe estudiar desde la relación que surge entre el entorno construido y dichas limitaciones. De esta manera, las personas pueden ser excluidas de una actividad por todo tipo de barreras, que surgen de la falta de coherencia del entorno con sus características físicas, sensoriales, cognitivas, sociales, psicológicas o económicas. Las consecuencias de este enfoque deberían ser el prestar más atención al diseño de todas las de espacios y servicios para que las personas no queden excluidas de ellas (Tyler, 2002).

\subsection{Exclusión por Diseño}

Dado que los productos, servicios y entornos con los que las personas interactúan son, en general, creados por el hombre, se puede decir que las personas que experimentan incompatibilidad al usarlos están excluidas por diseño (Coleman \& Myerson, 2001). La exclusión resulta si alguna de las demandas sensoriales, cognitivas o motoras de la tarea excede cualquiera de las habilidades correspondientes del usuario (Clarkson et al., 2013). La exclusión del diseño se ha definido como la incapacidad para utilizar un producto, servicio o instalación debido a que, con frecuencia, durante el proceso de diseño, no se han tenido en cuenta las necesidades de las personas que experimentan deficiencias motoras, sensoriales y cognitivas (BS 7000-6, 2005). Esta exclusión a menudo surge cuando los diseñadores, al tomar decisiones de diseño, no tienen en cuenta las capacidades funcionales del usuario final (Keates \& Clarkson, 2003). Cada decisión de diseño tiene el potencial de incluir o excluir clientes (Waller et al., 2013). Por tanto, la exclusión del diseño no se produce por casualidad; se produce por negligencia, falta de conocimiento y datos adecuados (Cassim et al., 2007).

Dada la disminución de la funcionalidad debido al proceso de envejecimiento (como el incremento en el aumento de las limitaciones motoras, visuales, auditivas, cognitivas o de salud), es común que la brecha entre las habilidades personales y las demandas ambientales se amplíe (Rogers et al., 1998; Seidel et al., 2009). En consecuencia, muchas personas mayores y/o con alguna discapacidad pueden verse privadas del uso de productos o servicios, y también de la participación en las actividades de la vida social como resultado de la falta de compatibilidad entre las demandas del entorno (productos y servicios) y sus propias capacidades cambiantes (Clarkson et al., 2013; Coleman \& Myerson, 2001). Coleman (2006) sugiere que es necesario reducir y eliminar la exclusión del diseño no solo desde una

ACE, 16 (4.7) CC BY-ND 3.0 ES | UPC Barcelona, España | Título del artículo escrito en mayúsculas y minúsculas, si es demasiado largo debe ajustarlo a dos líneas como máximo. DOI: http://dx.doi.org/10.5821/ace.16.47.9691 
perspectiva social (mejorar la calidad de vida de las personas mayores y/o con alguna discapacidad), sino también para el crecimiento empresarial a través de nuevos y mejores productos y servicios.

\subsection{Diseño Inclusivo}

Una de las filosofías básicas que subyace a este documento es la del Diseño Inclusivo, en la medida en que debemos pensar en un enfoque de diseño para las ciudades desde una perspectiva inclusiva. El diseño inclusivo no es un nuevo género de diseño ni una especialidad separada. Es un enfoque general del diseño en el que los diseñadores se aseguran de que sus productos, servicios y espacios respondan a las necesidades de la audiencia más amplia posible, independientemente de su edad o capacidad (Design Council, 2008). En la Norma Británica BS 7000-6 (2005) el diseño inclusivo se define como el diseño de productos y / o servicios de uso cotidiano para que sean accesibles y usables por la mayor cantidad de personas con diversas habilidades dentro de una amplia gama de situaciones sin necesidad de adaptaciones o diseños especiales. Sin embargo, una característica clave del diseño inclusivo es aumentar el grupo objetivo de un producto o servicio, pero sin comprometer los objetivos comerciales de ganancias y satisfacción del cliente (Coleman et al., 2003).

Un ejemplo clásico de aplicación de estos principios es el caso del transporte público en el Reino Unido. Mismo que en los últimos treinta años ha visto la introducción exitosa de autobuses accesibles que tienen pisos bajos y / o sistemas de arrodillamiento; así como pasamanos de colores contrastantes para facilitar su identificación a personas con alguna discapacidad visual. Este diseño de autobús ha permitido un acceso más fácil a los ancianos, los padres con niños pequeños, así como a los usuarios de sillas de ruedas (Balcombe et al., 2004). La importancia de este diseño se muestra en un estudio que sugirió que la introducción de estos autobuses junto con un pase gratuito ha llevado a un uso generalizado de los servicios de autobús por parte de personas mayores de 60 años y personas con alguna discapacidad (Schmöcker et al., 2008). Sin embargo, todavía hay espacio para más investigación y un uso generalizado de los principios filosóficos del Diseño Inclusivo para el mejoramiento de las ciudades. Por ejemplo, una investigación encargada por Age UK mostró que por día más de 800 personas mayores sufren una caída en un autobús y casi dos millones estaban preocupados por caerse mientras utilizaban el servicio de autobus (Department for Transport, 2012). Asimismo, se reporta que algunos usuarios de sillas de ruedas con frecuencia enfrentan problemas debido a que los espacios para sillas de ruedas están ocupados con frecuencia por carritos de bebé (Transport for all, 2014).

\section{Usabilidad y Diseño de Servicios, más allá de la Accesibilidad}

La usabilidad es un término utilizado en el campo de la ergonomía que se define como la "medida en que un producto puede ser utilizado por personas específicas para lograr objetivos específicos con eficacia, eficiencia y satisfacción en un contexto de uso específico" (ISO 9241-11, 1998), donde "la eficacia es la precisión y la integridad con la que los usuarios logran los objetivos especificados"; eficiencia "es la cantidad de recursos gastados en relación con la precisión e integridad con la que los usuarios logran los objetivos"; y la satisfacción "es la ausencia de molestias y actitudes positivas hacia el uso del producto". Cabe destacar que este concepto también define implícitamente lo que es la comodidad.

Por su parte, el término accesibilidad es conocido en muchas esferas sociales y áreas de aplicación como el Diseño y la Arquitectura. Dicho concepto se refería inicialmente a la relación entre las capacidades y las exigencias del diseño del entorno físico. Accesibilidad referida al cumplimiento de normas o estándares oficiales en términos físicos (Iwarsson \& Ståhl, 2003). Sin embargo, hoy en día

ACE, 16 (4.7) CC BY-ND 3.0 ES | UPC Barcelona, España | Título del artículo escrito en mayúsculas y minúsculas, si es demasiado largo debe ajustarlo a dos líneas como máximo. DOI: http://dx.doi.org/10.5821/ace.16.47.9691 
es un término más orientado a la usabilidad, que se define como la usabilidad de un producto, servicio, entorno o instalación por personas con un amplio rango de capacidades (BS 18477, 2010).

La accesibilidad orientada más hacia la usabilidad resulta de extrema utilidad cuando se piensa en aspectos de movilidad. Como ejemplo, se puede pensar en la existencia de rampas en las aceras cuya presencia podría ser considerada como una característica de accesibilidad. Sin embargo, será necesario evaluar y diseñar si dicha rampa podría ser utilizada con facilidad por el mayor rango de usuarios posibles. Esta situación invita a reflexionar no solo en la presencia de la rampa sino en características de inclinación, dimensiones, información y presencia o no de elementos que impidan su uso.

Además de la coherencia entre los aspectos físicos de los espacios y las características motrices de los usuarios, también surgen otros elementos de vital importancia y que se relacionan con aspectos cognitivos y organizacionales. Uno de ellos es la naturaleza, la claridad y la calidad de la información que se provee a un usuario para mejorar su interacción con el medio. Una calle que garantice la seguridad de los transeúntes, por ejemplo, debe estar acompañada de información cuyo diseño sea coherente con las capacidades sensoriales y cognitivas de quienes reciben dicha información. Si esto es así, no solo se garantiza el acceso a dicha información, sino que hará que la interacción del usuario con la calle sea más efectiva y segura, reduciendo los riesgos en espacios de alta accidentalidad como los cruces e intersecciones.

Otra ventaja que presenta el uso del concepto de accesibilidad desde la perspectiva de la usabilidad, es su idoneidad para ser aplicada, además de la infraestructura, los espacios y los objetos, en otros escenarios como los servicios. Dada su naturaleza compleja, los servicios se constituyen en terreno fértil para el uso del concepto de accesibilidad desde el ámbito de la usabilidad. La literatura sobre servicios se ha centrado en las características del servicio, que con frecuencia se comparan con las características de los productos; donde los servicios se caracterizan por su intangibilidad, inseparabilidad, heterogeneidad y carácter efímero (Zeithaml et al., 1985).

\subsection{Diseño de Servicios}

Un enfoque de diseño que es de utilidad para el diseño de mejores ciudades es el Diseño de Servicios, en la medida en que aporta una perspectiva integral de las condiciones presentes en estos contextos. Esto es evidente, por ejemplo, en el caso de los servicios de transporte en donde se ha encontrado que las barreras para el uso del autobús por parte de las personas mayores se extienden más allá de la interacción física con el vehículo. De manera similar, las posibles soluciones también pueden estar fuera del diseño del vehículo, por ejemplo, el comportamiento de los conductores o la mejora de la información del servicio. Por tanto, una perspectiva de Diseño de Servicios puede permitir una visión más amplia del usuario dentro del sistema de autobús.

El Diseño de Servicios tiene como objetivo innovar o mejorar servicios que sean útiles, utilizables y deseables desde la perspectiva del usuario, y eficientes y efectivos desde la perspectiva de las organizaciones (Mager \& Sung, 2011; Moritz, 2005). En consecuencia, sugiere que el Diseño de Servicios brinda varios beneficios a la experiencia del usuario final cuando se aplica a sectores como el comercio minorista, la banca, el transporte y la atención médica (Stickdorn, 2010b). Pero también se ha definido como un enfoque estratégico que ayuda a los proveedores de servicios a posicionar mejor sus ofertas de servicios (Mager \& Sung, 2011). En el ámbito de los servicios públicos, se ha señalado como un enfoque que tiene menos que ver con la competencia y la contestabilidad y más con la reducción de la brecha entre lo que hacen las organizaciones y lo que los usuarios esperan o necesitan (Parker \& Heapy, 2006). Este reconocimiento de la importancia del usuario sugiere que el diseño de servicios se relaciona adecuadamente con el enfoque de Ergonomía que respalda este

ACE, 16 (4.7) CC BY-ND 3.0 ES | UPC Barcelona, España | Título del artículo escrito en mayúsculas y minúsculas, si es demasiado largo debe ajustarlo a dos líneas como máximo. DOI: http://dx.doi.org/10.5821/ace.16.47.9691 
artículo. Stickdorn (2010a) ha definido cinco principios básicos que pueden utilizarse como marco filosófico para trabajar en Diseño de Servicios, mismos que se describen en la Tabla 1.

Tabla 1. Principios del Pensamiento de Diseño de Servicios

\begin{tabular}{|l|l|}
\hline Principio & Descripción \\
\hline 1. Centrado en las personas & $\begin{array}{l}\text { Los servicios deben [analizarse] y experimentarse a través de los ojos del } \\
\text { usuario(s). }\end{array}$ \\
\hline 2. Co-creativos & $\begin{array}{l}\text { Todos los actores involucrados deben estar incluidas en el proceso de diseño } \\
\text { del servicio. }\end{array}$ \\
\hline 3. Secuenciación & $\begin{array}{l}\text { El servicio debe visualizarse como una secuencia de comportamientos } \\
\text { interrelacionados. }\end{array}$ \\
\hline 4. Evidenciar & Los servicios intangibles deben visualizarse en términos de artefactos físicos. \\
\hline 5. Holístico & Se debe considerar todo el entorno [y elementos] de un servicio. \\
\hline
\end{tabular}

Dado que los servicios se caracterizan como entidades intangibles, uno de los aportes más relevantes del Diseño de Servicios es hacer tangible lo intangible (Kimbell, 2009; Meroni \& Sangiorgi, 2011). Para este enfoque de diseño es importante evidenciar la información transformándola en entidades físicas - para hacer tangible y visible lo intangible - y para ello los diseñadores de servicios utilizan una serie de herramientas y técnicas para visualizar el proceso del servicio (Kimbell, 2011; Mager, 2009; Polaine et al., 2013; Stickdorn \& Schneider, 2010). El uso de herramientas de visualización es una de las prácticas centrales en el Diseño de Servicios y puede afirmarse como uno de sus fundamentos (Segelström \& Holmlid, 2009). Existen una serie de herramientas de visualización como los "customer journey maps", "stakeholders maps" y "service blueprints", entre otros. Estos últimos se describen en el siguiente apartado.

\section{Herramientas útiles para el diseño de mejores ciudades}

Esta sección describe tres herramientas que se consideran de utilidad para la evaluación y el diseño de ciudades inclusivas, seguras y sostenibles. Dos de estas herramientas provienen de la Ergonomía y el diseño Inclusivo y la tercera tiene su origen el enfoque de Diseño de servicios. Dichas herramientas se seleccionaron para su descripción puesto que servirán para la presentación del estudio de caso que se describe en el punto 6 de este artículo.

\subsection{Análisis de tareas y Teoría Demanda-Capacidad}

La Ergonomía como disciplina científica y como profesión utiliza una serie de métodos y herramientas para el análisis y la intervención en las interacciones entre los seres humanos y otros elementos dentro de un sistema. Sin duda, el "análisis de tareas" ocupa un lugar especial dentro del repertorio de métodos en Ergonomía. Mismo que ha sido utilizado por los ergónomos desde finales de la década de los 60s (Stanton, 2006). Kirwan y Ainsworth (1992) definen el análisis de tareas como un estudio de lo que se requiere que un operador (o equipos de personas) haga en términos de acciones o procesos cognitivos, para lograr una meta del sistema. Por su parte, Shepherd y Stammers (2005) lo definen como un término para describir las actividades relacionadas con el examen del desempeño humano en los sistemas, tanto desde la perspectiva del comportamiento del ser humano como de los factores que dan forma a ese desempeño.

En otras palabras, el análisis de tareas es un método que se utiliza para dividir una tarea en componentes útiles de "tamaño más pequeño", lo cual proporciona una mejor comprensión sobre la profundidad y complejidad de las tareas. Esto puede ayudar a comprender la forma en que las personas trabajan dentro de un sistema; así como comprender la forma en que las personas

ACE, 16 (4.7) CC BY-ND 3.0 ES | UPC Barcelona, España | Título del artículo escrito en mayúsculas y minúsculas, si es demasiado largo debe ajustarlo a dos líneas como máximo. DOI: http://dx.doi.org/10.5821/ace.16.47.9691 
interactúan con todos los elementos del sistema. El análisis de tareas permite identificar áreas de oportunidad para el diseño o rediseño de dichos elementos del sistema a fin de hacerlos más compatibles con las necesidades, capacidades y limitaciones de las personas que los utilizan.

Además de analizar cada una de las tareas, diseñar para la inclusión requiere conocimiento y datos sobre las capacidades, limitaciones, necesidades y aspiraciones de los usuarios (Langdon et al., 2013) porque cuando interactúan con un producto o servicio, generalmente se les requiere que utilicen sus capacidades para percibir, pensar y actuar (Clarkson et al., 2007). La literatura sobre Diseño Inclusivo ha definido las siguientes categorías que son útiles para evaluar la capacidad que demanda un producto para usarlo (Clarkson et al., 2013):

- Visión: la capacidad de usar el color y el brillo de la luz para detectar objetos, discriminar entre diferentes superficies o ver el detalle en una superficie;

- $\quad$ Audición: la capacidad de discriminar tonos o habla específicos del ruido ambiental y de saber de dónde provienen los sonidos;

- Pensamiento: la capacidad de procesar información, mantener la atención, comunicarse, almacenar y recuperar recuerdos y seleccionar respuestas y acciones adecuadas;

- Alcance y destreza: la capacidad de alcanzar con una o ambas manos por encima de la cabeza, hacia el frente, hacia el suelo, realizar una manipulación fina de los dedos y transportar objetos.

- Movilidad: la capacidad de moverse, subir escalones y mantener el equilibrio de forma independiente.

Con base en la Ergonomía (o Factores Humanos), y en los conceptos de capacidad del usuario y demanda de producto, Persad et al. (2007) desarrollaron la Teoría de Demanda-Capacidad (que se muestra esquemáticamente en la Figura 1). Este modelo sirve como una herramienta analítica para evaluar la compatibilidad entre el usuario y el producto. El uso de este marco permite comprender y describir la demanda que un producto, servicio, y/o ambiente, o elemento de un sistema ejerce sobre las personas.

Figura 1. Interacción entre capacidades del usuario y demanda del producto

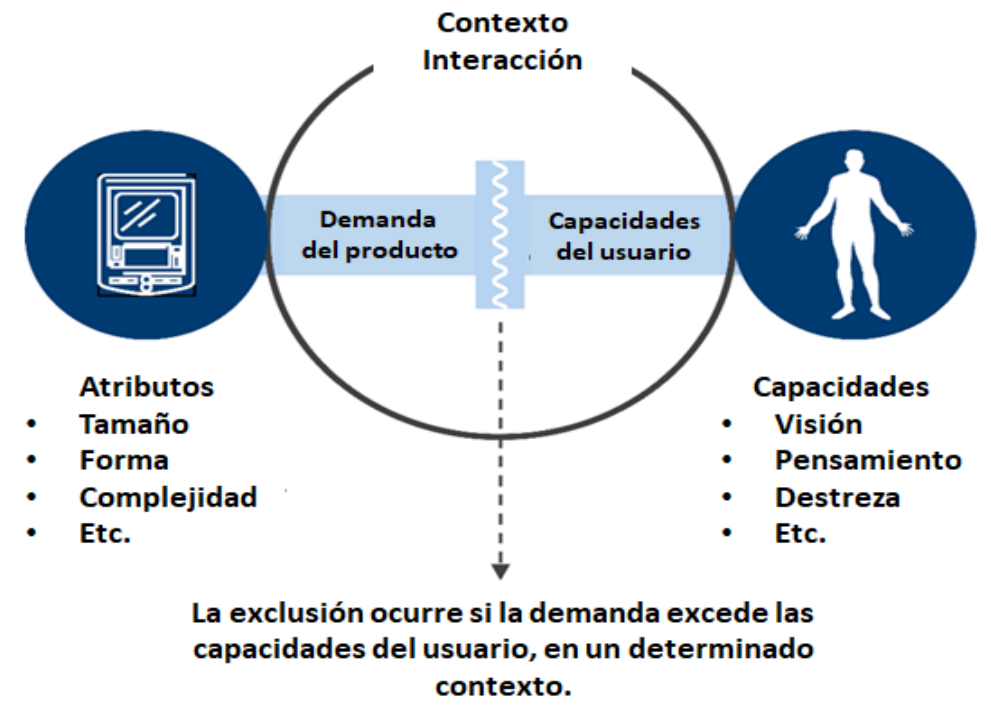

Fuente: Adaptada y traducida de Persad et al., 2007

Un punto que resulta relevante en esta búsqueda por involucrar herramientas de la Ergonomía y el Diseño Inclusivo en el mejoramiento de las ciudades, tiene que ver con que, tanto el Análisis de tareas como la Teoría de la Demanda-Capacidad presentan la ventaja de ajustarse a diversas escalas y tipos de problemáticas. El rediseño de un parquímetro, el rediseño de una estación de autobús, o el

ACE, 16 (4.7) CC BY-ND 3.0 ES | UPC Barcelona, España | Título del artículo escrito en mayúsculas y minúsculas, si es demasiado largo debe ajustarlo a dos líneas como máximo. DOI: http://dx.doi.org/10.5821/ace.16.47.9691 
rediseño de la operación del servicio del metro, por ejemplo, parecen tener poco en común, considerando sus diferentes propósitos y niveles de complejidad. Sin embargo, en los tres casos tenemos dos elementos esenciales que son la interacción usuario-sistema y, los requerimientos de las tareas. En relación al primero, el Análisis de tareas nos permitiría describir los momentos de interacción entre el usuario y el sistema para identificar áreas de mejora. En el caso del segundo, la Teoría de Demanda-Capacidad nos permitiría comprender la coherencia entre los requerimientos de las tareas y las características del usuario y con ello establecer mejoras en la interacción. En este sentido, podemos afirmar que cualquier interacción entre usuario y sistema puede ser descrita y analizada con estos dos lentes, lo que las hace herramientas versátiles que pueden contribuir a mejorar los procesos de desarrollo de los sistemas presentes dentro de ese mega-sistema que es la ciudad.

\subsection{Service blueprinting: una técnica para visualizar y comunicar el conocimiento sobre el servicio}

Como se mencionó líneas arriba, el Diseño de Servicios utiliza herramientas y técnicas de representación para visualizar los componentes no tangibles de un servicio. Si bien existe una amplia gama de herramientas de visualización disponibles, una de las más útiles para describir la investigación sobre el servicio es el blueprint del servicio.

La creación de blueprints de servicios es un método introducido por Shostack (1982, 1984) y desarrollado después por Kingman-Brundage para visualizar los procesos de los servicios (Fließ \& Kleinaltenkamp, 2004). Como lo define el Design Council (2013): Un blueprint del servicio es una representación visual detallada del servicio total a lo largo del tiempo, que muestra el viaje del usuario, todos los diferentes puntos y canales de contacto, así como las partes detrás de escena de un servicio que lo hace funcionar.

Fließ y Kleinaltenkamp (2004) describen un blueprint de servicio como una imagen bidimensional de un proceso de servicio. El eje horizontal representa las acciones realizadas cronológicamente por el cliente del servicio y el prestador del servicio, y el eje vertical diferencia entre diferentes áreas de actuación. Bitner, Ostrom y Morgan (2008) indican que un blueprint típico de servicio comprende los siguientes componentes, que se ilustran aquí con un ejemplo de servicio de autobús:

- Acciones del cliente, por ejemplo, pagar la tarifa;

- Acciones visibles de los empleados, por ejemplo, la interacción con el conductor;

- Acciones no visibles de los empleados, por ejemplo, el procesamiento adicional del dinero recaudado por el conductor;

- $\quad$ Procesos de soporte, por ejemplo, sistemas contables; y

- $\quad$ Evidencia física, por ejemplo, el ticket de cliente.

Estas áreas de acción están separadas por "líneas", que Fließ \& Kleinaltenkamp (2004) definen de la siguiente manera: “línea de interacción” que separa el área de acción del cliente del área de acción del proveedor, lo que representa las interacciones directas entre el cliente y proveedor. Por encima de la "línea de interacción", encontramos actividades, elecciones e interacciones realizadas por el cliente, por ejemplo, la interacción con el conductor. La "línea de visibilidad" se divide entre acciones visibles e invisibles para el cliente. Por encima de la "línea de visibilidad", se muestran las acciones y decisiones llevadas a cabo por los empleados de la oficina principal - lo que el pasajero puede ver . La "línea de interacción interna" distingue entre las actividades de los empleados de primera línea y los de la oficina administrativa. Los procesos de soporte, que son necesarios para apoyar la prestación del servicio, se llevan a cabo debajo de la "línea de interacción interna", por ejemplo, lo que el pasajero no puede ver, sistemas contables, cómo se paga al conductor, suspensión del autobús. La Tabla 2 muestra los componentes y la estructura de un blueprint típico.

ACE, 16 (4.7) CC BY-ND 3.0 ES | UPC Barcelona, España | Título del artículo escrito en mayúsculas y minúsculas, si es demasiado largo debe ajustarlo a dos líneas como máximo. DOI: http://dx.doi.org/10.5821/ace.16.47.9691 
Tabla 2. Componentes de un blueprint de servicios

\begin{tabular}{l|l}
\hline Evidencia física & \\
\hline Acciones del usuario & Línea de interacción \\
\hline Escenario/ contacto visible / Acciones de los empleados & Línea de visibilidad \\
\hline Detrás del escenario / Contacto invisible / Acciones de los & Línea de interacción interna \\
\hline empleados & \\
\hline Procesos de apoyo &
\end{tabular}

Un blueprintes una herramienta extremadamente útil para el diseño de servicios (Polaine et al., 2013), porque es más preciso que las definiciones verbales y menos sujeto a interpretaciones (Shostack, 1984). Los blueprints de servicio son relativamente simples y sus representaciones gráficas son fáciles de asimilar, utilizar e incluso adaptar para todos los actores involucrados (clientes, gerentes y empleados de primera línea) para satisfacer las diferentes necesidades de cada servicio. El blueprint de servicio puede aplicarse para comprender y diseñar experiencias de servicio ideales y puede proporcionar un medio para que los diseñadores de servicios contrasten el servicio ideal del cliente y el servicio existente real de la empresa.

Los blueprints de servicios también se pueden utilizar como un método para generar ideas para un nuevo servicio (blueprints para la innovación de servicios), o como un proceso de análisis para mejorar un servicio existente (blueprints para la mejora del servicio) (Polaine et al., 2013). Según Segelström (2009) cuando se mejora un servicio, los blueprints son útiles para ayudar a comprender el contexto, así como para identificar oportunidades de diseño, mientras que la herramienta de escenarios futuros se adapta mejor al crear un servicio completamente nuevo.

\section{Blueprint para un servicio de transporte inclusivo - estudio de caso}

Este apartado describe el desarrollo de un blueprint para un servicio inclusivo, que ayuda a visualizar y comunicar conocimientos sobre las características reales del servicio de autobús en el Área Metropolitana de Guadalajara, México; así como las brechas entre lo que hacen los proveedores de servicios y lo que los usuarios jóvenes y adultos mayores esperan o necesitan según sus habilidades y limitaciones.

Este blueprint de servicios inclusivos fue desarrollado por Aceves-González (2014) utilizando principios, métodos y herramientas de la Ergonomía y los enfoques de Diseño Inclusivo y Diseño de Servicios, mismos que han sido descritos en los apartados previos. Este blueprint se basa en datos que han sido publicados previamente sobre el mismo servicio de transporte (C. Aceves-González et al., 2015; Carlos Aceves-González et al., 2016).

La intención al construir este blueprint era traducir los datos emanados de la investigación científica en conocimientos útiles y comunicarlos a los actores involucrados del servicio de autobús de una manera gráfica. Como se indicó anteriormente, la creación del blueprint de servicios es una técnica de visualización que se utiliza para estos fines y es una práctica fundamental en el diseño de servicios. Además, se debe destacar la falta de herramientas que respalden el uso efectivo de los datos del usuario en el proceso de diseño (Dong et al., 2013; Nickpour \& Dong, 2011).

\subsection{Formato del blueprint de servicios inclusivos}

ACE, 16 (4.7) CC BY-ND 3.0 ES | UPC Barcelona, España | Título del artículo escrito en mayúsculas y minúsculas, si es demasiado largo debe ajustarlo a dos líneas como máximo. DOI: http://dx.doi.org/10.5821/ace.16.47.9691 
El blueprint de servicios inclusivos se diseñó teniendo en cuenta el formato típico de un blueprint, incluido el escenario y el detrás del escenario dividido por la línea de visibilidad. Puede verse en la Figura 2 que la sección detrás del escenario retuvo exactamente los mismos componentes de un blueprint típico. Se aplicaron dos adaptaciones a la sección del escenario. Primero, la sección de evidencia física se eliminó de la parte superior y se integró en la misma sección de puntos de contacto y actividades del personal.

La intención era concentrar el enfoque en las acciones del usuario en la parte superior del plano, pero también se consideró que la evidencia física podría integrarse en esa sección a fin de simplificar el plan para que las personas interesadas lo comprendan mejor. En segundo lugar, la línea de interacción se utilizó para representar el nivel de dificultad en el uso del servicio por parte de personas jóvenes y adultos mayores, y la brecha entre lo que los usuarios necesitan y lo que hacen las organizaciones de servicios de transporte.

Finalmente, a medida que se preparaba el blueprint para los interesados en el servicio, se prestó especial atención al uso de imágenes y colores en el diseño del mismo. Según Segelström (2009), existe una diferencia en la naturaleza de las visualizaciones utilizadas en un blueprint según quién debe verlas: personas externas o el equipo de diseño.

Las visualizaciones para clientes o actores involucrados suelen ser más sencillas y estéticamente más atractivas. En consecuencia, con la intención de aumentar la simplicidad y el atractivo del blueprint, se representaron las acciones del usuario y algunos puntos de contacto mediante el uso de imágenes. Los colores se utilizaron para hacerlo más atractivo, pero también para diferenciar claramente las secciones dentro del plano. Los criterios de diseño que incluyen simplicidad, visibilidad, legibilidad y legibilidad y armonía de color se consideraron al usar colores e imágenes.

\subsection{Diseño del contenido del blueprint de servicios inclusivos}

En cuanto al contenido, el servicio de autobús se dividió en siete etapas, las cuales se han definido con base en la revisión de la literatura (Nickpour et al., 2012; Tyler, 2002), mismas que inician cuando el usuario está en casa y concluyen cuando arriba a su destino. De manera similar, el blueprint incluye las acciones del usuario que se han identificado a partir de un análisis de tareas con base en los estudios reportados por Aceves-González et al. (2015; 2016).

Solo se incluyeron acciones generales para cada etapa porque demasiados detalles en un blueprint pueden disminuir su capacidad para proporcionar una visión general que sea de utilidad (Polaine et al., 2013). Los puntos de contacto, las acciones del conductor y la evidencia física se definieron con base en estudios previos (Aceves-González et al., 2014; C. Aceves-González et al., 2015; Carlos AcevesGonzález et al., 2016).

El blueprint está diseñado y presentado en tamaño AO. Dado este tamaño, el plano se presenta primero en una versión completa en miniatura (Figura 2) y luego se presenta un acercamiento a algunas de sus partes para permitir su lectura (Figura 3).

ACE, 16 (4.7) CC BY-ND 3.0 ES | UPC Barcelona, España | Título del artículo escrito en mayúsculas y minúsculas, si es demasiado largo debe ajustarlo a dos líneas como máximo. DOI: http://dx.doi.org/10.5821/ace.16.47.9691 


\section{ACE Architecture, City and Environment}

Figura 2. Blueprint de servicios inclusivos

Blueprint para el diseño de servicios inclusivos
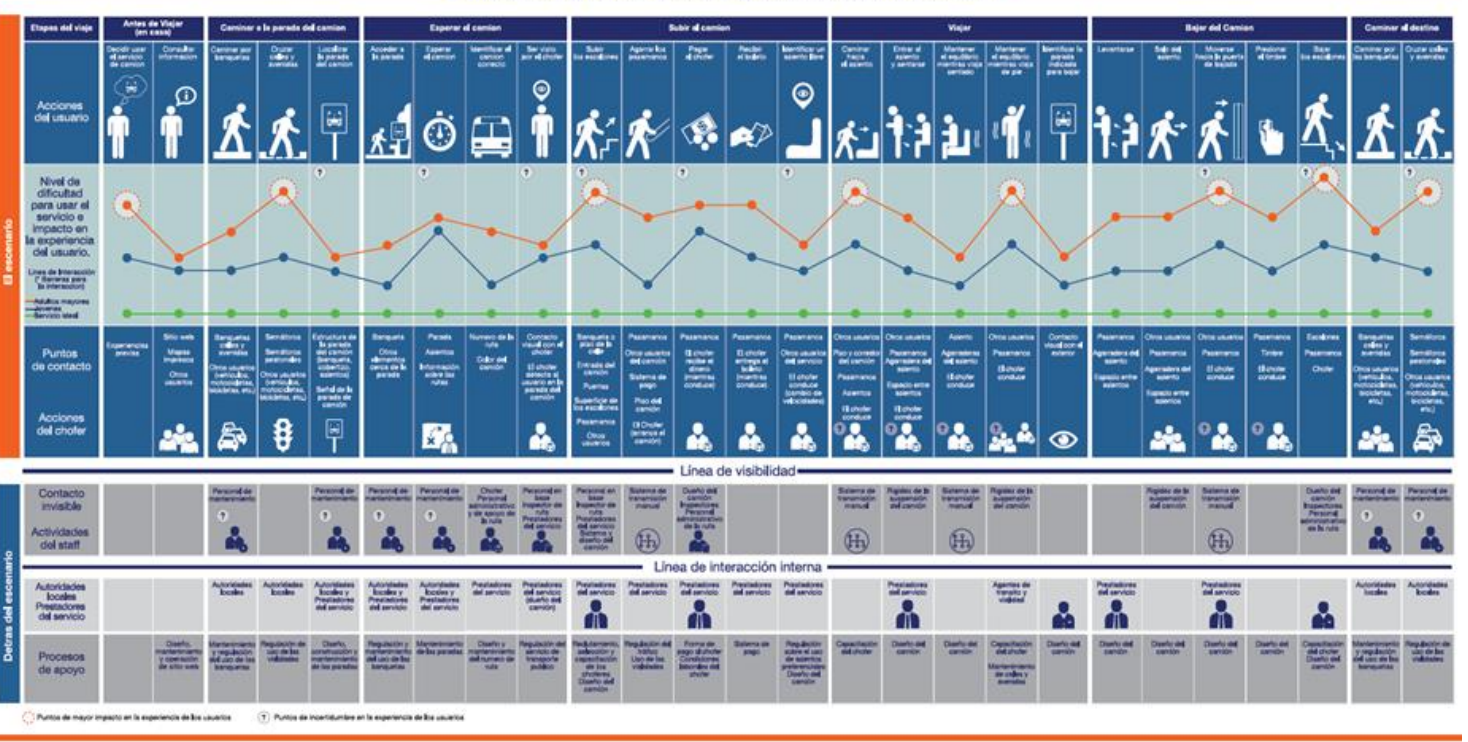

Fuente: Versión completa del blueprint en miniatura (Aceves-González, 2014)

Figura 3. Sección con primeras etapas del recorrido del usuario (Aceves-González (2014)

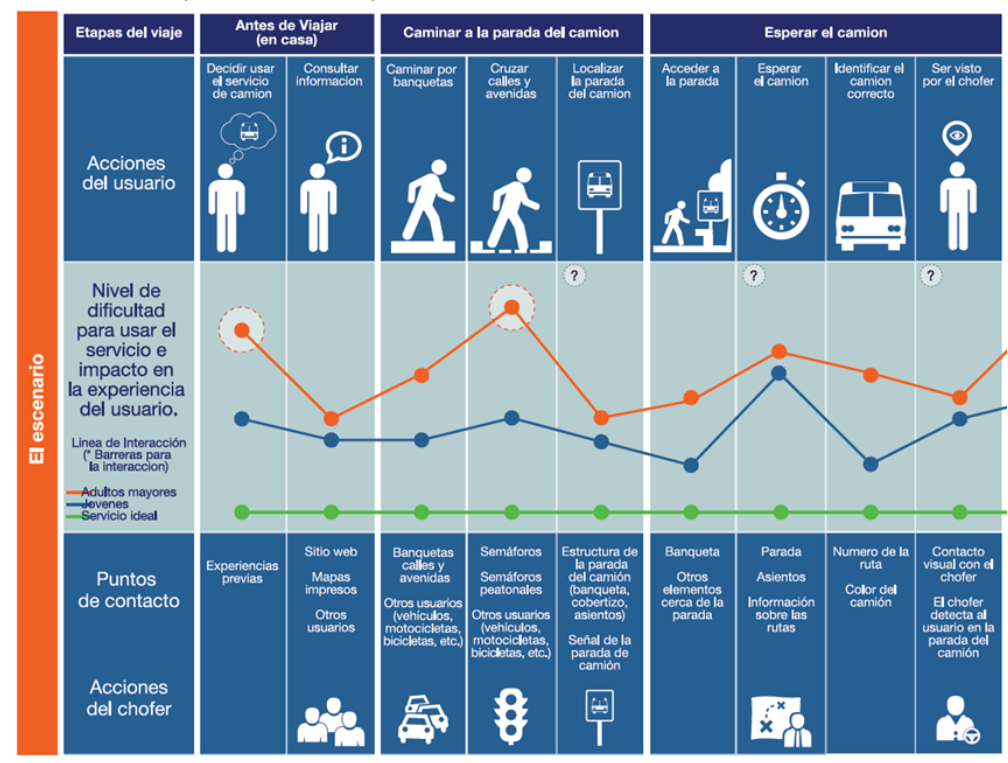

Fuente: Aceves-González, 2014

\subsection{Características clave del blueprint de servicios inclusivos}

El desarrollo del blueprint de servicios inclusivos tuvo como objetivo ayudar a visualizar y comunicar a los actores involucrados información sobre las características reales del servicio de autobús, pero especialmente las brechas entre lo que hacen los proveedores de servicios y lo que los usuarios jóvenes y mayores esperan o necesitan de acuerdo con sus capacidades y limitaciones. La figura 3 muestra la versión completa del blueprint. Una observación detallada permite identificar las siguientes características clave del blueprint inclusivo: 
- Ilustra la diferencia entre la experiencia y las necesidades de las personas jóvenes y mayores al utilizar el servicio de autobús,

- Visualiza diferencias en el nivel de dificultad para utilizar el servicio para jóvenes y adultos mayores,

- $\quad$ Proporciona un medio para contrastar el servicio ideal y la experiencia del usuario dentro del servicio existente,

- $\quad$ Ayuda a demostrar visualmente cómo la brecha entre las habilidades personales y las demandas ambientales se hace más amplia para las personas mayores.

- Sugiere que, al abordar las necesidades de las personas mayores para mejorar el servicio, también se podrían considerar las necesidades de los más jóvenes,

- Muestra los puntos de interacción asociados con un mayor nivel de dificultad, particularmente para usuarios mayores; y por tanto proporciona un conjunto de prioridades para diseñar un servicio de autobús más inclusivo.

- $\quad$ Además, proporciona las principales barreras que deben abordarse para reducir las dificultades para los usuarios y aumentar la aceptación del servicio.

\subsection{Evaluación y uso del blueprint de diseño de servicios inclusivos}

El proceso de elaboración del blueprint representó una serie de desafíos, desde comprender la demanda de capacidad que requiere el servicio en cada punto de interacción; analizar datos para determinar la capacidad del usuario; estimar el nivel de dificultad; y finalmente presentar los datos de una manera útil y deseable, como sugirieren Clarkson y Coleman (2013) y Nickpour y Dong (2011). Desde su desarrollo, este blueprint ha sido evaluado a través de talleres con diversos usuarios (Aceves-González, 2018), además de utilizarse en actividades de docencia. Su uso en estas actividades ayuda generalmente a captar el interés de los participantes en el uso de la herramienta. En general, los resultados indican que el blueprintes una herramienta útil, fácil de usar y atractiva. La singularidad de este blueprint es su incorporación explícita de la inclusión puesto que un blueprint típico solo describe las acciones del cliente, pero no considera la diversidad de los usuarios y sus habilidades, limitaciones, necesidades y expectativas.

Es importante mencionar que, si bien este enfoque ha sido aplicado en el contexto del servicio de autobús en Guadalajara, el blueprint descrito en este artículo puede ser aplicable para analizar y diseñar servicios inclusivos relacionados con el cuidado de la salud, turismo, finanzas e incluso servicios digitales, por mencionar algunos, en otros contextos alrededor del mundo.

Como se mencionó al inicio del presente texto, garantizar el bienestar de los habitantes de las ciudades no solo es un paso dentro de los procesos de desarrollo de ciudades más sostenibles y seguras, sino que parece ser uno de sus puntos esenciales. Esto nos lleva a la necesidad de desarrollar ciudades inclusivas, proveedoras de una alta calidad de vida para todos sus habitantes, en donde su infraestructura y servicios correspondan a los requerimientos y características de todos los usuarios. Así pues, la perspectiva que provee el blueprint de Diseño de Servicios Inclusivos, donde se reconoce la amplia diversidad de características presentes en los usuarios de un servicio, puede ser muy importante para re pensar los servicios y demás prestaciones que ofrecen las ciudades. Herramientas como el blueprint de Servicios Inclusivos y demás alternativas de diseño centrado en los seres humanos, constituyen una alternativa idónea para la consecución de un futuro con una calidad de vida más prometedora para las generaciones venideras.

ACE, 16 (4.7) CC BY-ND 3.0 ES | UPC Barcelona, España | Título del artículo escrito en mayúsculas y minúsculas, si es demasiado largo debe ajustarlo a dos líneas como máximo. DOI: http://dx.doi.org/10.5821/ace.16.47.9691 


\section{Conclusiones}

Este documento es una respuesta ante la necesidad de diseñar y construir ciudades más inclusivas, seguras y sostenibles. Con la intención de aportar ante esa necesidad, el objetivo de este artículo fue mostrar el uso de alternativas para lograrlo, entre ellas, el uso de la disciplina científica de la ergonomía, con su enfoque centrado en las personas y en las actividades humanas; el diseño inclusivo con su filosofía de considerar las capacidades y limitaciones, necesidades y expectativas de los diversos usuarios; y el diseño de servicios con su perspectiva de análisis de la interacción de los componentes de los sistemas. Con la presentación de esta disciplina y enfoques de diseño, sus herramientas y métodos, así como su aplicación en un caso de estudio, es posible identificar diversos beneficios y el potencial implícito y explicito para el diseño y construcción de mejores ciudades.

Sin duda, uno de los mayores beneficios es el enfoque de sistemas que nos ofrece la Ergonomía, mismo que permite tener una visión integral para el análisis de problemas/sistemas complejos. Por ejemplo, en el caso de la movilidad o el trasporte público permite el análisis de 1) todos los actores involucrados en la prestación del servicio (usuarios, conductores de autobuses, operadores de servicios, autoridades locales, etc.); 2) todos o la mayoría de los componentes del servicio (información, diseño de autobuses, rutas, conductores, otros usuarios del servicio o de la vía pública, etc.); 3) todas las interacciones del usuario a lo largo de las etapas de un viaje puerta a puerta (antes del viaje, caminando hasta la parada del autobús, esperando el autobús, etc.), y de igual manera las interacciones de otros involucrados con el servicio. Como resultado, se puede obtener información relevante que permita comprender las necesidades de los usuarios y todos los actores involucrados (por ejemplo, los conductores de autobuses). Asimismo, permite la identificación y comprensión de los factores que podrían causar fallas u obstáculos para la movilidad o la prestación del servicio de transporte público.

Otro de los beneficios para la construcción de ciudades sostenibles, seguras e inclusivas surge de la filosofía de la Ergonomía de estar centrada en las personas, aunado al enfoque del Diseño Inclusivo sobre la diversidad de las personas. Puesto que en la medida que los espacios y servicios estén diseñados considerando las capacidades, limitaciones, necesidades y expectativas de las personas, se garantiza un uso más seguro y una mayor demanda de uso de los mismos. Esto traducido en materia de transporte público garantizaría que una mayor cantidad de usuarios deseará usarlo, lo cual daría como resultado un servicio de transporte más sostenible. Además, con efectos positivos en términos sociales, económicos y ambientales.

Por el contrario, la falta de consideración de las características y necesidades de las personasciudadanos conlleva a la exclusión, cada decisión de diseño tiene el potencial de incluir o excluir. La exclusión del diseño no se produce por casualidad; sino por negligencia, ignorancia y falta de información y datos adecuados. En ese sentido es de particular relevancia tener en cuenta la información que pueda ser proporcionada por usuarios y/o ciudadanos, lo cual permite la recopilación de datos únicos que comprenden componentes personales que serían difíciles de identificar (o comprender) para personas fuera de ese grupo de usuarios, como los profesionales del Diseño, el Urbanismo o la Arquitectura. Por ejemplo, en el caso del servicio de autobús, los actores involucrados (conductores, operadores de autobuses, fabricantes de autobuses, diseñadores y reguladores) es poco probable que experimenten directamente por sí mismos los desafíos que enfrentan los diversos pasajeros al usar el servicio de autobús. La mayoría de los involucrados (excepto los conductores) no están en contacto directo con pasajeros y les puede resultar difícil identificar dónde los elementos del servicio impactan a cada grupo de usuarios (niños, adolescentes, mujeres, mujeres embarazadas, adultos mayores, y personas con alguna discapacidad, por mencionar solo algunos).

ACE, 16 (4.7) CC BY-ND 3.0 ES | UPC Barcelona, España | Título del artículo escrito en mayúsculas y minúsculas, si es demasiado largo debe ajustarlo a dos líneas como máximo. DOI: http://dx.doi.org/10.5821/ace.16.47.9691 
A pesar de que el enfoque se aplicó en un caso de estudio de servicio de autobuses en México, representa una oportunidad para explorar y proporcionar conocimientos que podrían ser transferibles para el Diseño de ciudades sostenibles, seguras e inclusivas en otros contextos, particularmente en países de bajo y mediano ingreso. Este artículo contribuye a la discusión de la utilidad de integrar principios de Ergonomía y estos enfoques de diseño y sugiere que ambos dominios de diseño, ciudadanos, empresarios y gobiernos podrían ser receptores de los beneficios de dicha integración.

\section{Agradecimientos}

Los autores de este articulo agradecemos a los pares revisores, puesto que con sus acertados comentarios se ha logrado mejorar el contenido y edición de este documento.

\section{Autoría}

Ambos autores contribuyeron en todas las etapas de construcción de este artículo. El primer autor participó principalmente en la conceptualización y escritura del trabajo. El segundo autor ha participado en la conceptualización, diseño y escritura del trabajo.

Conflicto de intereses: Los autores declaran que no hay conflicto de intereses.

\section{Bibliografía}

Aceves-Gonzalez, C. (2014). The application and development of inclusive service design in the context of a bus service [Loughborough University]. Recuperado de https://dspace.lboro.ac.uk/2134/16265

Aceves-Gonzalez, C. (2018). Ergonomics and inclusive service design: Exploring possibilities for its application in a developing world city. En Di Bucchianico G., Kercher P. (eds) Advances in Design for Inclusion. AHFE 2017. Advances in Intelligent Systems and Computing (pp. 369-377). DOI: https://doi.org/10.1007/978-3-319-60597-5 35

Aceves-Gonzalez, C., Cook, S., \& May, A. (2014). Inclusive service design: In search of better services. En M. Soares \& F. Rebelo (Eds.), Advances in Ergonomics in Design, Usability \& Special Populations. Part /// (pp. 10-19). Published by AHFE Conference (c) 2014.

Aceves-González, C., Cook, S., \& May, A. (2015). Bus use in a developing world city: Implications for the health and well-being of older passengers. Journal of Transport and Health, 2(2), 308-316. DOI: https://doi.org/10.1016/j.jth.2015.04.001

Aceves-González, Carlos, May, A., \& Cook, S. (2016). An observational comparison of the older and younger bus passenger experience in a developing world city. Ergonomics, 59(6), 840-850. DOI: https://doi.org/10.1080/00140139.2015.1091513

ADA. (1990). Americans with Disabilities Act. Department of Justice, Civil Rights Division.

Balcombe, R., Mackett, R., Paulley, N., Preston, J., Shires, J., Itheridge, H., Wardman, M., \& White, P. (2004). The demand for public transport: a practical guide, TRL Report. TRL 593.

ACE, 16 (4.7) CC BY-ND 3.0 ES | UPC Barcelona, España | Título del artículo escrito en mayúsculas y minúsculas, si es demasiado largo debe ajustarlo a dos líneas como máximo. DOI: http://dx.doi.org/10.5821/ace.16.47.9691 
Bibri, S. E., \& Krogstie, J. (2017). Smart sustainable cities of the future: An extensive interdisciplinary literature review. Sustainable Cities and Society, 31, 183-212. DOI: https://doi.org/10.1016/j.scs.2017.02.016

Bitner, M. J., Ostrom, A. L., \& Morgan, F. N. (2008). Service Blueprinting: A Practical Technique for Service Innovation. Management Review, 50(3), 66-95. DOI: https://doi.org/10.2307/41166446

BS 18477. (2010). BS 18477:2010. Inclusive service provision - Requirements for identifying and responding to consumer vulnerability. BSI. UK.

BS 7000-6. (2005). Design Management Systems: Managing Inclusive Design (Vol. 3). BSI. UK.

Calderón, C., Salas, H. \& Ávila, P. (2020). La insostenibilidad de los desarrollos de vivienda de interés social en México: una aproximación desde el pensamiento de diseño. Caso de estudio: Tlajomulco de Zúñiga, Jalisco. ACE: Architecture, City and Environment, 14(42), 8256. DOI: http://dx.doi.org/10.5821/ace.14.42.8256

Cassim, J., Coleman, R., Clarkson, J., \& Dong, H. (2007). Why Inclusive Design? In R. Coleman, J. Clarkson, H. Dong, \& J. Cassim (Eds.), Design for Inclusivity. A Practical Guide to Accessible, Innovative and User-Centred Design. Gower.

CEPAL (2010). El desarrollo sostenible en América Latina y el Caribe: Tendencias, avances y desafíos en materia de consumo y producción sostenibles, minería, transporte, productos químicos y gestión de residuos. Informe para la decimoctava sesión de la Comisión sobre el Desarrollo Sostenible de las Naciones Unidas. Naciones Unidas. Santiago de Chile, Chile.

Clarkson, J., \& Coleman, R. (2013). History of Inclusive Design in the UK. Applied Ergonomics, 46(B), 235-247. DOI: https://doi.org/10.1016/j.apergo.2013.03.002

Clarkson, J., Coleman, R., Hosking, I., \& Waller, S. (2007). Inclusive Design Toolkit (First Edit). University of Cambridge.

Clarkson, J., Dong, H., \& Keates, S. (2003). Quantifying design exclusion. En J Clarkson, S. Keates, R. Coleman, \& C. Lebbon (Eds.), Inclusive Design: Design for the whole population. Springer Verlag

Clarkson, John, Waller, S., \& Cardoso, C. (2013). Approaches to estimating user exclusion. Applied Ergonomics, 46(B), 304-310. DOI: https://doi.org/10.1016/j.apergo.2013.03.001

Coleman, R. (2006). From Margins to mainstream: why inclusive design is better design. The Ergonomics Society Annual Lecture.

Coleman, R., Lebbon, C., Clarkson, J., \& Keates, S. (2003). Introduction: From Margins to Mainstream. En John Clarkson, R. Coleman, S. Keates, \& C. Lebbon (Eds.), Inclusive Design. Designing for the whole population. Springer-Verlag.

Coleman, R., \& Myerson, J. (2001). Improving Life Quality by Countering Design Exclusion. Geronthecnology, 1(2), 88- 102. Recuperado de https://journal.gerontechnology.org/archives/43-46-1$\underline{\text { PB.pdf }}$

Crimmins, E. M. (2004). Trends in the health of the elderly. Annual Review of Public Health, 25, 79-98. DOI: https://doi.org/10.1146/annurev.publhealth.25.102802.124401

ACE, 16 (47) CC BY-ND 3.0 ES | UPC Barcelona, España | Título del artículo escrito en mayúsculas y minúsculas, si es demasiado largo debe ajustarlo a dos líneas como máximo. DOI: http://dx.doi.org/10.5821/ace.16.47.9691 
DDA. (1995). Disability Discrimination Act. The Stationery Office.

Department for Transport. (2012). Transport Solutions for older People. Information Resource for Local Authorities (Issue February, pp. 1-30). Department for Transport. Recuperado de https://www.gov.uk/government/uploads/system/file/4478/transport-solutions-for-older-people.pdf

Design Council. (2008). Inclusive Design Education Resource. Design Council. Recuperado de http://www.designcouncil.info/inclusivedesignresource/index.html

Design Council. (2013). Introducing Design Methods. Recuperado de http://www.designcouncil.org.uk/news-opinion/introducing-design-methods

Dong, H., McGinley, C., Nickpour, F., \& Cifter, A. S. (2013). Designing for designers: Insights into the knowledge users of inclusive design. Applied Ergonomics, 46(b), 1-8. DOI: https://doi.org/10.1016/j.apergo.2013.03.003

Dul, J., Bruder, R., Buckle, P., Carayon, P., Falzon, P., Marras, W. S., Wilson, J. R., \& van der Doelen, B. (2012). A strategy for human factors/ergonomics: developing the discipline and profession. Ergonomics, 55(4), 377-395. DOI: https://doi.org/10.1080/00140139.2012.661087

Fließ, S., \& Kleinaltenkamp, M. (2004). Blueprinting the service company. Journal of Business Research, 57(4), 392-404. DOI: https://doi.org/10.1016/S0148-2963(02)00273-4

Habib, F., \& Shokoohi, A. (2009). Classification and resolving urban problems by means of fuzzy approach. World Academy of Science, Engineering and Technology, 36(June), 896-903. DOI: https://doi.org/10.5281/zenodo.1085388

Haslam, R., \& Waterson, P. (2013). Ergonomics and Sustainability. Ergonomics, 56(3), 343-347. DOI: https://doi.org/10.1080/00140139.2013.786555

IEA. (2000). International Ergonomics Asociation. Definition of Ergonomics. Recuperado de https://iea.cc/

ISO 9241-11. (1998). Ergonomic Requirements for Office Work with Visual Display Terminals (VDTS) Part 11: Guidance on Usability. International Standards Organisation.

Iracheta, A. (2020). La ciudad que quisiéramos después de COVID-19. ACE: Architecture, City and Environment, 15(43), 9512. DOI: http://dx.doi.org/10.5821/ace.15.43.9512

Iwarsson, S., \& Ståhl, A. (2003). Accessibility, usability and universal design--positioning and definition of concepts describing person-environment relationships. Disability and Rehabilitation, 25(2), 57-66. DOI: https://doi.org/10.1080/dre.25.2.57.66

Jordán R., Riffo L., \& Prado A. (2017). Desarrollo sostenible, urbanización y desigualdad en América Latina y el Caribe. Dinámicas y desafíos para el cambio estructural. Naciones Unidas. Santiago de chile, Chile.

Keates, S., \& Clarkson, J. (2003). Countering design exclusion: bridging the gap between usability and accessibility. Universal Access in the Information Society, 2, 215-225. DOI: https://doi.org/10.1007/s10209-003-0059-5 
Keivani, R. (2010). A review of the main challenges to urban sustainability. International Journal of Urban Sustainable Development, 1(1-2), 5-16. DOI: https://doi.org/10.1080/19463131003704213

Kimbell, L. (2009). The turn to service design. En Julier, G. and Moor, L. (editors), Design and Creativity: Policy, Management and Practice, Oxford: Berg, pp. 157-173.

Kimbell, L. (2011). Designing for Service as One Way of Designing Services. International Journal, 5(2), 41-52. Recuperado de http://www.ijdesign.org/index.php/IJDesign/article/view/938/345

Kirwan, B., \& Ainsworth, L. (1992). A guide to task analysis: the task analysis-working group. CRC Press.

Kuhlman, T., \& Farrington, J. (2010). What is sustainability? Sustainability, 2(11), 3436-3448. DOI: https://doi.org/10.3390/su2113436

Langdon, P., Johnson, D., Huppert, F., \& Clarkson, P. J. (2013). A framework for collecting inclusive design data for the UK population. Applied Ergonomics, 46(B), 318-324. DOI: https://doi.org/10.1016/j.apergo.2013.03.011

Mager, B. (2009). Service Design as an Emerging Field. In S. Miettinen \& M. Koivisto (Eds.), Designing Services with Innovative Methods. University of Art and Design Helsinki.

Mager, B., \& Sung, T. (2011). Special issue editorial: Designing for services. International Journal of Design, 5(2), 2-4. Recuperado de http://www.jjdesign.org/index.php/IJDesign/article/view/994/341

Meroni, A., \& Sangiorgi, D. (2011). Design for Services. Gower.

Moray, N. (1995). Ergonomics and the global problems of the twenty-first century. Ergonomics, 38(8), 1691-1707. DOI: https://doi.org/10.1080/00140139508925220

Moritz, S. (2005). Service Design. Practical Access to an Evolving Field.

Nickpour, F., \& Dong, H. (2011). Designing Anthropometrics! Requirements Capture for Physical Ergonomic Data for Designers. The Design Journal, 14(1), 92-111. Recuperado de http://www.ingentaconnect.com/content/bloomsbury/dsgj/2011/00000014/00000001/art00006

Nickpour, F., Jordan, P. W., \& Dong, H. (2012). Inclusive Bus Travel - A Psychosocial Approach. In Patrick Langdon, J. Clarkson, P. Robinson, J. Lazar, \& A. Heylighen (Eds.), Designing Inclusive Systems (pp. 1323). Springer London. DOI: https://doi.org/10.1007/978-1-4471-2867-0

Parker, S., \& Heapy, J. (2006). The Journey to the Interface. How Public Service Design Can Connect Users to Reform. (p. 117). Demos. Recuperado de http://hmtreasury.gov.uk/d/cypreview2006 wiltshirecouncil2.pdf

Persad, U., Langdon, P., \& Clarkson, J. (2007). Characterising user capabilities to support inclusive design evaluation. Universal Access in the Information Society, 6(2), 119-135. DOI: https://doi.org/10.1007/s10209-007-0083-y

Polaine, A., Løvlie, L., \& Reason, B. (2013). Service Design. From Insight to Implementation. Rosenfeld Media, LLC.

ACE, 16 (4.7) CC BY-ND 3.0 ES | UPC Barcelona, España | Título del artículo escrito en mayúsculas y minúsculas, si es demasiado largo debe ajustarlo a dos líneas como máximo. DOI: http://dx.doi.org/10.5821/ace.16.47.9691 
Richardson, M., Maspero, M., Golightly, D., Sheffield, D., Staples, V., \& Lumber, R. (2017). Nature: a new paradigm for well-being and ergonomics. Ergonomics, 60(2), 292-305. DOI: https://doi.org/10.1080/00140139.2016.1157213

Rogers, W. a., Meyer, B., Walker, N., \& Fisk, A. D. (1998). Functional Limitations to Daily Living Tasks in the Aged: A Focus Group Analysis. Human Factors: The Journal of the Human Factors and Ergonomics Society, 40(1), 111-125. DOI: https://doi.org/10.1518/001872098779480613

Roth, I.-J. (2012). Just Cities: The World's Problems Need Urban Solutions. In Friedrich-Ebert-Stiftung, Global Policy and Development. Vol. Perspectiv (Issue February, p. 6 p.).

Schmöcker, J.-D., Quddus, M. a., Noland, R. B., \& Bell, M. G. H. (2008). Mode choice of older and disabled people: a case study of shopping trips in London. Journal of Transport Geography, 16(4), $257-267$. DOI: https://doi.org/10.1016/j.jtrangeo.2007.07.002

Segelström, F. (2012, September). Communicating through visualizations: Service designers on visualizing user research. In Conference Proceedings ServDes. 2009; DeThinking Service; ReThinking Design; Oslo Norway 24-26 Nov. 2009 (No. 059, pp. 175-185). Linköping University Electronic Press.

Segelström, F., \& Holmlid, S. (2009). Visualizations as tools for research: Service Designers on visualizations. En Nordes, Nordic Design Research Conference, 1-9. Recuperado de http://www.nordes.org/opj/index.php/n13/article/view/53

Seidel, D., Crilly, N., Matthews, F. E., Jagger, C., Clarkson, P. J., \& Brayne, C. (2009). Patterns of Functional Loss Among Older People: A Prospective Analysis. Human Factors: The Journal of the Human Factors and Ergonomics Society, 51(5), 669-680. DOI: https://doi.org/10.1177/0018720809353597

Shepherd, A., \& Stammers, R. (2005). Tasl Analysis. In J. Wilson, \& N. Corlett, (Eds.). (2005). Evaluation of human work. CRC press.

Shostack, G. (1982). How to design a service. European Journal of Marketing, 16, 49-63. Recuperado de http://www.ingentaconnect.com/content/mcb/007/1993/00000016/00000001/art00005

Shostack, G. (1984). Designing services that deliver. Harvard Business Review, 62(January-February), 133-139.

Smith, D. (1990). Human factors and aging: An overview of research needs and application opportunities. Human Factors, 32(5), 509-526. DOI: https://doi.org/10.1177/001872089003200502

Stanton, N. A. (2006). Hierarchical task analysis: Developments, applications, and extensions. Applied Ergonomics, 37(1 SPEC. ISS.), 55-79. DOI: https://doi.org/10.1016/j.apergo.2005.06.003

Stickdorn, M. (2010a). 5 Principles of Service Design Thinking. In M. Stickdorn \& J. Schneider (Eds.), This is Service Design Thinking: Basics, Tools. Cases. BIS Publishers.

Stickdorn, M. (2010b). Definitions: Service Design as an Interdisciplinary Approach. In M. Stickdorn \& J. Schneider (Eds.), This is Service Design Thinking: Basics, Tools, Cases (p. 373). BIS Publishers.

Stickdorn, M., \& Schneider, J. (2010). This Is Service Design Thinking: Basics, Tools, Cases. BIS Publishers.

ACE, 16 (4.7) CC BY-ND 3.0 ES | UPC Barcelona, España | Título del artículo escrito en mayúsculas y minúsculas, si es demasiado largo debe ajustarlo a dos líneas como máximo. DOI: http://dx.doi.org/10.5821/ace.16.47.9691 
Thatcher, A. (2013). Green ergonomics: Definition and scope. Ergonomics, 56(3), 389-398. DOI: https://doi.org/10.1080/00140139.2012.718371

Thatcher, A., Waterson, P., Todd, A., \& Moray, N. (2018). State of Science: ergonomics and global issues. Ergonomics, 61(2), 197-213. DOI: https://doi.org/10.1080/00140139.2017.1398845

The Lancet. (1999). The spectrum of disability. The Lancet, 354(9180), 693. DOI: https://doi.org/10.1016/S0140-6736(99)90152-6

Transport for all. (2014). Accessing the service. Recuperado de http://www.transportforall.org.uk/public/bus/

Tyler, N. (2002). Accessibility and the bus system: From concepts to practice. Thomas Telford Services Ltd.

United Nations. (2002). World Population Ageing 1950-2050. Department of Economic and Social Affairs, Population Division

United Nations. (2009). Population Ageing and Development. Department of Economic and Social Affairs, Population Division

Waller, S., Bradley, M., Hosking, I., \& Clarkson, P. J. (2013). Making the case for inclusive design. Applied Ergonomics, 46(B), 1-7. DOI: https://doi.org/10.1016/j.apergo.2013.03.012

WCED. (1987). World commission on environment and development. Our common future.

WHO. (2007). Global Age-friendly Cities: A Guide. WHO. Recuperado de http://www.who.int/ageing/age friendly cities guide/en/

WHO. (2011). World Report on Disability. World Health Organization. Geneva.

WHO. (2012). Disability A66/12. Provisional Agenda. World Health Organization. Recuperado de http://apps.who.int/gb/ebwha/pdf files/WHA66/A66 12-en.pdf?ua=1

Wilson, J. R. (2000). Fundamentals of ergonomics in theory and practice. Applied Ergonomics, 31(6), 557-567. DOI: https://doi.org/10.1016/S0003-6870(00)00034-X

Wilson, J. R. (2014). Fundamentals of systems ergonomics/human factors. Applied Ergonomics, 45(1), 5-13. DOI: https://doi.org/10.1016/j.apergo.2013.03.021

Winchester L. (2006). Desafíos para el desarrollo sostenible de las ciudades en América Latina y El Caribe. Revista EURE, 32(96), 7-25. DOI: http://dx.doi.org/10.4067/S0250-71612006000200002

Wogalter, M. S., Hancock, P. A., \& Dempsey, P. G. (1998). On the description and definition of Human Factors/Ergonomics. Proceedings of the Human Factors and Ergonomics Society, 1(February 2015), 671-674. DOI: https://doi.org/10.1177/154193129804201001

Wolf, K. L. (2003). Ergonomics of the City: Green Infrastructure and Social Benefits. Engineering Green Proceedings of the 2003 National Urban Forest Conference, 2003.

Zeithaml, V., Parasuraman, A., \& Berry, L. (1985). Problems and strategies in services marketing. The Journal of Marketing, 49(2), Spring, 33-44. DOI: https://doi.org/10.2307/1251563

ACE, 16 (4.7) CC BY-ND 3.0 ES | UPC Barcelona, España | Título del artículo escrito en mayúsculas y minúsculas, si 23 es demasiado largo debe ajustarlo a dos líneas como máximo. DOI: http://dx.doi.org/10.5821/ace.16.47.9691 